\title{
Neurotechnology and Psychiatric Biomarkers
}

\author{
William J. Bosl \\ Harvard Medical School, \\ Children's Hospital Boston Informatics Program, \\ Boston University School of Medicine Behavioral Neuroscience Program,
}

USA

\section{Introduction}

Neuroscience as a scientific discipline has enjoyed enormous growth and success in the past decade. Some have called the early $20^{\text {th }}$ century the golden age of physics, the latter half of the $20^{\text {th }}$ century a period when the genomic revolution blossomed and predict that the early $21^{\text {st }}$ century will be a period when brain sciences achieve remarkable success. While understanding the basic mechanisms of the brain and how they relate to thought and behavior may be the foundation for applications to medicine, there is a great need for technological innovation if more than academic results are to be achieved. The need for neurotechnology is already great. Traumatic brain injury and damaged limbs require prosthetic devices that can be controlled in some way by willful volition. Ideally, direct connections between thought and action are desirable to restore natural functions. Mental health is a branch of medicine that has long been relegated to a secondary status within medicine. The reasons for this may be many, but they certainly include the difficulty of understanding and measuring brain activity in a quantitative way and relating those measurements to behavior and cognitive activity. As healthcare costs continue to spiral out of control in both developed and developing regions of the world, the need for engineers to become involved in neuroscience and neurotechnology research and development has never been greater. Innovative engineering ideas, with a view toward practical application and affordable cost have much to contribute to clinical applications of brain science. A key contribution of neuroengineering will be innovative methods for quantitative measurement of brain activity and mapping of those measurements to behavior and thought. The term psychiatric biomarkers will be used here in this broad sense to indicate quantitative measurements of the brain and the algorithms necessary to interpret them in psychiatric or psychological descriptions or diagnoses.

One important way in which psychiatric biomarkers differ from other physiological biomarkers is in that the mapping from biomarker to symptom or disease is much more complex. A biomarker for a specific cancer, for example, may be a gene mutation that is in some way directly involved in the disease progression itself. The relationship between biomarker and the manifestation of interest - cancer, in this case - is rather simple and direct. That is not to say that the gene or the gene expression patterns are simple to find, but only that the conceptual relationship between the marker and disease is simple to understand. In the case of psychiatric biomarkers, the phenomena of interest, thought and behavior, are complex, emergent phenomena of brain neural activity. The relationship 
between neural firing patterns and the communication deficits that are clearly evident in a person with a mental disorder is not at all clear, even if we posit that all thought is indeed dependent upon neural activity. The relationship in this case is somewhat like the relationship between letters of the alphabet and a metaphor in great literature. Certainly metaphors depend on spelling and grammar, but the concept is much more than spelling and grammar. Similarly, the complex patterns of neural activity that distinguish the way a person with autism responds to someone speaking directly to them from someone considered "normal" are quite complex.

Normal and abnormal behavior are differentiated by subtle, complex patterns of activity that a trained expert observes or discovers through systematic diagnostic tests. If brain function and behavior are mirrors of each other, as is commonly accepted (Cowan and Kandel, 2001; Hyman, 2007; Kandel, 1998; Singh and Rose, 2009), then biomarkers of mental disorders may be hidden in subtle, complex patterns of neurobiological data. There is a growing realization that the neurophysiological mechanisms that underlie brain function cannot be understood by pure reduction to physiological causes (Stam, 2005; Ward, 2003). The dynamics of the brain is inherently nonlinear, exhibiting emergent dynamics such as chaotic and transiently synchronized phenomena that may be central to understanding the mind-brain relationship (Varela et al., 2001) or the 'dynamic core' (Le Van Quyen, 2003). The behaviors and thoughts that characterize mental dysfunction may be emergent phenomena or complex patterns of physiological processes, especially neural processes. For example, major depression or the communication deficits present in a child with autism are emergent phenomena that reflect complex patterns in brain function that differ from some sociallydefined norm. The task of the neuroengineer is to create new technology to measure and interpret the patterns of brain activity that connect brain measurements to observed behavioral patterns.

A key challenge in cognitive neuropsychiatry is to discover the neural correlates underlying behavior. To be clinically useful, these discoveries must be accompanied by technology that enables brain activity to be measured and interpreted safely, inexpensively and easily. The explosive growth of neuroimaging studies that link functional brain activity to behavior promises exciting opportunities for measuring nonlinear brain activity that may indicate abnormalities or allow response to therapy to be monitored. While several imaging modalities are available for neuroscience research, most have significant limitations that prohibit their use as routine clinical tools. Cost and ease of use are essential qualities for clinically useful tools, which may not be as important or relevant in a scientific research context. Neuroengineers must be cognizant of these constraints when considering the intended use of the technology.

Measurements of brain electrical activity with electroencephalography (EEG) have long been a valuable source of information for neuroscience research, yet this rich resource may be under-utilized for clinical applications in neurology and psychiatry (Niedermeyer, 2003; Niedermeyer and Lopes da Silva, 2005). To fully exploit this data, methods for discovering subtle nonlinear patterns and deeper understanding of the relationship between emergent signal features, neurophysiology and behavior are needed. Near infrared spectroscopy (NIRS) has recently been introduced as a safe, portable alternative for measuring blood oxygen level dependent (BOLD) response in infants (Irani et al., 2007; Muehlemann et al., 2008). One of the primary advantages of NIRS, like EEG, is that it is safe for all ages, relatively inexpensive and portable. As a new brain-imaging tool, much remains to be discovered about the value and limitations of NIRS as a clinical instrument. In addition, 
coupling EEG and NIRS may have some advantages for clinical use and remains to be explored by researchers. Many of the advances in non-invasive functional brain measurement are being driven by the brain computer interface community, where mobility and cost requirements limit the technologies that can be adopted (Dornhege, 2007). Neuropsychology and cognitive neuropsychiatry can learn from this community, while adapting the methods to the particular needs of behavioral, affective and cognitive assessment.

In this chapter some relevant information concerning our current understanding of complex network organization and implications for finding neural correlates of behavior will be reviewed with goal of motivating engineers to consider contributing their skills to developing new neurotechnology. Considerable attention will be given to EEG measurements as one of the most promising technologies for clinical application to neuropsychiatry. Novel methods for extracting information from EEG signals are beginning to appear, taking advantage of advances in the physics of nonlinear systems and signals, complex network theory and machine learning algorithms. The need for innovative neurotechnology to meet the need for mental and neurological healthcare in developing regions of the world is great, but the promise is even greater. The primary goal of this chapter is to provide information to enable researchers interested in brain disorders and mental health to become involved in creating innovative neurotechnology for clinical use.

\section{The brain as a complex system}

\subsection{Complex networks}

The human brain contains on the order of $10^{11}$ neurons and more than $10^{14}$ synaptic connections (Kandel et al., 2000). Although sparsely connected, each neuron is within a few synaptic connections of any other neuron (Buzsáki, 2006). This remarkable connectivity is achieved by a kind of hierarchical organization that is not fully understood in the brain, but is ubiquitous in nature, called scale-free or complex networks (Barabasi, 2009; Bassett and Bullmore, 2006; Ravasz and Barabasi, 2003). Complex networks are characterized by dense local connectivity and sparser long-range connectivity (Barabasi, 2009) that is fractal or selfsimilar at all scales.

Many brain disorders appear to be associated with abnormal brain connectivity that may vary between different regions and different scales (Bassett and Bullmore, 2009; Craddock et al., 2009; Noonan et al., 2009). Examples include autism (Belmonte et al., 2004; Noonan et al., 2009), schizophrenia (Raghavendra et al., 2009; Uhlhaas et al., 2008; Whittington, 2008), depression (Li et al., 2008; Sheline et al., 2009) and epilepsy (Douw et al., 2010; Percha et al., 2005). Methods for estimating neural connectivity or changes in neural connectivity might be effective diagnostic biomarkers for abnormal connectivity development that is associated with brain dysfunction. The electrical signals produced by neural networks are believed to contain information about the neural network structure on several scales in the vicinity of the EEG sensor (Raghavendra et al., 2009; Stam, 2005; Zavaglia et al., 2008).

Novel analysis methods from nonlinear systems theory are able to extract information from EEG signals that reflect the underlying network organization. System invariants will be encoded in the time series produced and measured by EEG sensors (Fuchs et al., 2007; Gao and Jin, 2009). Multiscale entropy (MSE) is one invariant measure of system dynamics that has been shown to particularly sensitive to changes in physiological systems (Costa et al., 2005b; Hu et al., 2009b; Takahashi et al., 2010), including mental disorders such as 
Alzheimer's Disease (Abasolo et al., 2006), schizophrenia (Takahashi et al., 2010), the effect of antipsychotic drugs (Takahashi et al., 2010) normal aging (Bruce et al., 2009) and autism spectrum disorders (Bosl et al., 2011).

A comparison of functional network properties using fMRI showed that children and young-adults' brains have similar "small-world" organization at the global level, but differ significantly in hierarchical organization and interregional connectivity (Supekar et al., 2009). The networks measured with fMRI in this study are those that are formed among correlated voxels, which may represent a different spatial scale from that measured by single EEG sensors. Transient or sustained generalized synchronization between EEG sensors is another measurement of functional connectivity in the brain. A simultaneous study of EEG and fMRI signals in patients with a degenerative type of epilepsy showed that in the non-myoclonus state, subtle abnormalities that were detected in EEG signals did not affect fMRI, suggesting that EEG measurements of connectivity may measure different connectivity or may be more sensitive to temporal synchronization that occurs on a time scale less than that of fMRI (on the order of one second).

Neuroscience has made great progress using linear methods of spectral (Fourier) analysis, it is likely that much more information is contained in the complex patterns of brain activity (see, for example, (Bruce et al., 2009). EEG may be under-utilized for clinical applications in neurology and psychiatry (Niedermeyer, 2003; Niedermeyer and Lopes da Silva, 2005) particularly now as new developments in complex systems and multivariate nonlinear time series analysis may allow previously unexplored information to be extracted from EEG signals (Kulisek et al., 2008; Mizuhara et al., 2005; Stam, 2005; Varela et al., 2001). Sensors such as EEGs measure the coordinated electrical response of many neurons to produce time series that reflect the dynamics of this complex system. In order to extract salient information from this data, methods appropriate for analyzing nonlinear time series are required. Although many useful techniques for nonlinear time series and system analysis have been developed in other disciplines (Bosl, 2000; Braha et al., 2006; Elnashaie and Grace, 2007; Holland, 1995; Stauffer, 2006), it is not immediately clear which are most appropriate for neuroscience research. Nevertheless, recent research results are quite promising.

The complexity of EEG signals was found in one study to be associated with the ability to attend to a task and adapt to new cognitive tasks; a significant difference in complexity was found between normal subjects and those with diagnosed schizophrenia (Li et al., 2008). Schizophrenic patients were found to have lower complexity than normal controls in some EEG channels and significantly higher interhemispheric and intrahemispheric cross mutual information values (another measure of signal complexity) than normal controls (Na et al., 2002).

Methods for chaotic signal and phase synchronization analysis arose from a need to rigorously describe physical phenomena that exhibited what was formerly thought to be purely stochastic behavior but was then discovered to represent complex, aperiodic selforganized dynamics (Pikovsky et al., 2001). The analysis of signal complexity and interaction between signals leading to transient synchronization may reveal information about local neural complexity and long-range communication between brain regions (Buzsáki, 2006; Stam, 2005; Varela et al., 2001).

The synchronization patterns of complex networks have been shown to be closely related to the topology of the network (Arenas et al., 2006) and are related to brain connectivity (Sakkalis et al., 2008). EEG signals are believed to derive from pyramidal cells aligned in parallel in the cerebral cortex and hippocampus (Sörnmo and Laguna, 2005), which act as 
many interacting nonlinear oscillators (Nunez and Srinivasan, 2006). As a consequence of the scale-free network organization of neurons, EEG signals exhibit complex system characteristics reflecting the underlying network topology, including transient synchronization between frequencies, short and long range correlations and crossmodulation of amplitudes and frequencies (Gans et al., 2009). A great deal of information about interrelationships in the nervous system likely remains hidden because the linear analysis techniques currently in use fail even to detect them (Drongelen, 2007).

\subsection{Mental processes as emergent phenomena}

Much of modern scientific medicine is reductionist, involving a search for ultimate basic causes of disease. This paradigm for scientific research follows naturally from the extraordinary success of physics in the last century, with its search for the fundamental laws of the universe. But that model is giving way to a new vision of the universe as a complex dynamical system, one in which fundamental laws may in fact be emergent properties of the system (Laughlin, 2005).

Emergent properties are those that result from the organization of individual parts and do not exist apart from the organizational whole. The saying that the whole is more than the sum of the parts is a description of an emergent property. The process of cell division, for example, is an emergent process that cannot be explained or studied using quantum mechanics - even though quantum mechanics is a good description of how atoms interact, and a cell is made up of many atoms, each obeying the fundamental physics of quantum theory. Similarly, the difference between a well-written high school essay for a college admissions committee and a Pulitzer Prize winning novel is not to be found in grammar and spelling, even though proper spelling and grammar are essential to the meaning of literature. Literature, genre and metaphor are emergent phenomena, more than words and sentences.

Developing neurotechnology, including devices and analysis methods, may be the most challenging subfield of biomedical engineering because the phenomenon of interest, a mental state or a complex set of behaviors that may indicate a diagnosis of a mental disorder, is an emergent phenomenon. Human behavior is controlled by the brain, which is ultimately a complex network of neurons that transmit electrochemical signals. Thought and behavior cannot be understood or measured by studying neurons (or genes) alone. Psychiatric biomarkers that focus on complex system properties may be the most informative measurements for assessing mental state. We now present a survey of complex system properties that can be computed from time series of brain electrical activity. Neural activity is electrochemical activity. Taking into account degradation due to the skull and scalp and the introduction of noise by the electronic sensors, EEG may be the most direct measurement of brain function that is possible. Thought might be considered an emergent phenomenon of neural electrical activity.

\section{Methods}

\subsection{Univariate measures}

Many different methods for computing the complexity of time series have been defined and used successfully to analyze EEG data (Chen et al., 2009; Kuusela et al., 2002). Sample entropy, a measure of nonlinear time series complexity, was significantly higher in certain regions of the right hemisphere in pre-term neonates that received skin-to-skin contact than 
in those that did not, indicating faster brain maturation (Scher et al., 2009). Sample entropy has also been used as a marker of brain maturation in neonates (de la Cruz et al., 2007) and was found to increase prenatally until maturation at about 42 weeks, then decreased after newborns reached full term (Zhang et al., 2009). A study of the correlation dimension (another measure of signal complexity) of EEG signals in healthy subjects showed an increase with aging, interpreted as an increase in the number of independent synchronous networks in the brain (Stam, 2005).

Intuitively, complexity is associated with structural richness, depth, patterns upon patterns, incorporating correlations over multiple spatio-temporal scales (Costa et al., $2005 b$ ). There is no consensus on a definition of complexity, but algorithms have been developed to attempt to give meaning to complexity. In the context of time series analysis, the concept of entropy is relevant. The use of entropy measures to describe the information content of time series began with the publication of Shannon's Mathematical Theory of Communication (Shannon and Weaver, 1949). In an intuitive sense, information is a measure of the difference in uncertainty before and after a measurement. In the context of time series, information is related to the predictability of the series. Entropy is a mathematical function of the probability that the next point in a sequence or time series will be a certain values, given the previous (Baddeley, 2000). Several different entropy measures can be defined algorithmically, including Shannon entropy, spectral entropy, approximate entropy, Lempel-Ziv complexity and sample entropy (Sabeti et al., 2009), each with certain advantages for particular time series characteristics (length, amount of noise, for example). The sample entropy has been used for a number of investigations of physiological signals. Changes in sample entropy appear to correlate with aging and pathological conditions in the context of cardiac health (Bruce et al., 2009; Costa et al., 2008; Norris et al., 2008) and for normal brain development (Zhang et al., 2009) and to distinguish certain mental disorders such as schizophrenia (Sabeti et al., 2009; Takahashi et al., 2010).

The multiscale entropy (MSE) analysis is one method for computing the complexity of a time series that builds on the sample entropy and expands the concept. It has been used to analyze a number of physiological processes (Costa et al., 2005b; Hornero et al., 2009; Norris et al., 2008; Takahashi et al., 2010). The multiscale entropy algorithm incorporates two steps. The first is a coarse-graining procedure that uses successive averaging of a time series to create new coarse-grained time series. For a window size $\tau, \tau=1,2, \ldots \mathrm{j}$, the $\mathrm{j}^{\text {th }}$ coarse-grain series, $\mathrm{y}_{\mathrm{j}}^{\tau}$, is computed by averaging non-overlapping windows:

$$
y_{j}^{\tau}=\frac{1}{\tau} \sum_{i=(j-1) \tau+1}^{j \tau} x_{i}
$$

where $x_{i}$ is the original time series of length $N$ and $\tau$ is the scale factor satisfying $1<\tau<N / \tau$. A schematic illustration of this process is shown in equation 2 .

\begin{tabular}{|c|c|c|c|c|c|c|c|c|c|}
\hline Scale 2 : & $x_{1}$ & $x_{2}$ & $x_{3}$ & $\mathrm{x}_{4}$ & $x_{5}$ & $x_{6}$ & $x_{7}$ & $x_{8}$ & $x_{k}=\left(s_{i}+s_{i+1}\right) / 2$ \\
\hline Scale 3 : & $\mathrm{y}_{1}$ & & $\mathrm{y}_{2}$ & & & $\mathrm{y}_{4}$ & & & $y_{k}=\left(s_{i}+s_{i+1}+s_{i+2}\right) / 3$ \\
\hline Scale 4: & & $z_{1}$ & & & & & & & $z_{k}=\left(s_{i}+s_{i+1}+s_{i+2}+s_{i+3}\right.$ \\
\hline
\end{tabular}


The coarse-graining method for extracting signal variability on different scales used by (Costa et al., 2005b) seems to be a heuristic procedure without any solid theoretical foundation. Other procedures can be substituted that may be justified on similar grounds. Perhaps the most immediate alternative would be to use the median rather than the mean value in each coarse graining step. This would have the effect of emphasizing the variability of the original signal rather than smoothing out such variability. Another procedure would be to select every kth point from the original series, where $\mathrm{k}$ is the desired scale, and use a pre-selected window size to compute an average value at the kth point. A systematic discussion and computational experiments have yet to be done for the coarse graining procedure that is central to the multiscale entropy algorithm.

The second step is to then compute the entropy of each of the coarse-grain time series $y^{\tau}$, using some entropy measure. The sample entropy is the most common entropy formulation to be used for analyzing physiological signals (Costa et al., 2005b). A useful variation to the original multiscale entropy algorithm uses the modified sample entropy defined in (Xie et al., 2008). The practical effect of using the modified sample entropy is the computed entropy values are more robust to noise and results are more consistent with short time series. In brief, the similarity functions $\mathrm{A}^{\mathrm{m}}$ and $\mathrm{B}^{\mathrm{m}}$ defined by equations (7) and (9) in (Xie et al., 2008) are computed for each coarse-grained time series defined in equation 1 . The modified multiscale entropy (mMSE) is then defined as the series of modified sample entropy values at each of the coarse grain scales. This method was used for complexity analysis of EEG time series as a biomarker for autism risk (Bosl, et al. 2011).

An alternative to the MSE is the scale dependent Lyapunov exponent (SDLE) algorithm described in (Gao, 2007; Gao et al., 2006). This measure of complexity is stable for short, noisy time series and reportedly is able to distinguish a number of different types of chaotic motion, including noise-induced chaos, stochastic oscillations and others, which entropy measures are not able to do. SDLE has not yet been used to analyze EEG signals in young children or infants. The SDLE algorithm is based on following the time evolution of all pairs of vectors in phase space that satisfy a given embedding restriction. This results in a rather straightforward algorithm for computing the SDLE. The SDLE is reportedly better at distinguishing noise from chaotic dynamics in time series. SDLE was shown to be a more effective measure of heart disease than sample entropy and MSE (Hu et al., 2009a). Similarly, SDLE was shown to be more effective in retrospectively identifying changes in EEG signal complexity just prior to the onset of epileptic seizures than MSE, but few other studies of SDLE with EEG time series have been done. This is a potentially promising measure to be investigated further.

\subsection{Detecting nonlinearity in time series}

Living systems exhibit a fundamental propensity to move forward in time. This property also describes physical systems that are far from an equilibrium state. For example, heat moves in only one direction, from hot to cold areas. In thermodynamics, this property is related to the requirement that all systems must move in the direction of higher entropy. Time irreversibility was found to be a characteristic of healthy human heart electrocardiogram (EKG) recordings and was shown to be a reliable way to distinguish between actual EKG recordings and model EKG simulations (Costa et al., 2008). EKG signals from patients with congestive heart disease were found to have lower time irreversibility indices than healthy patients (Costa et al., 2005a). Interestingly, time irreversibility of EEG signals has been associated with epileptic regions of the brain and this measure has been 
proposed as a biomarker for seizure foci (Gautama et al., 2003). Time irreversibility may be used as a practical test for nonlinearity in a time series.

As an illustration, a time irreversibility index $\left(t_{\text {rev }}\right)$ was computed for different resolutions of the EEG time series using the algorithm of (Costa et al., 2008). The third column of Figure 1 shows $t_{\text {rev }}$ for several different linear and nonlinear time series. Of particular note is that only the sine wave time series and both random time series have nearly zero irreversibility indices, while the index for the nonlinear logistic series and the representative EEG signal are both nonzero on all scales shown.
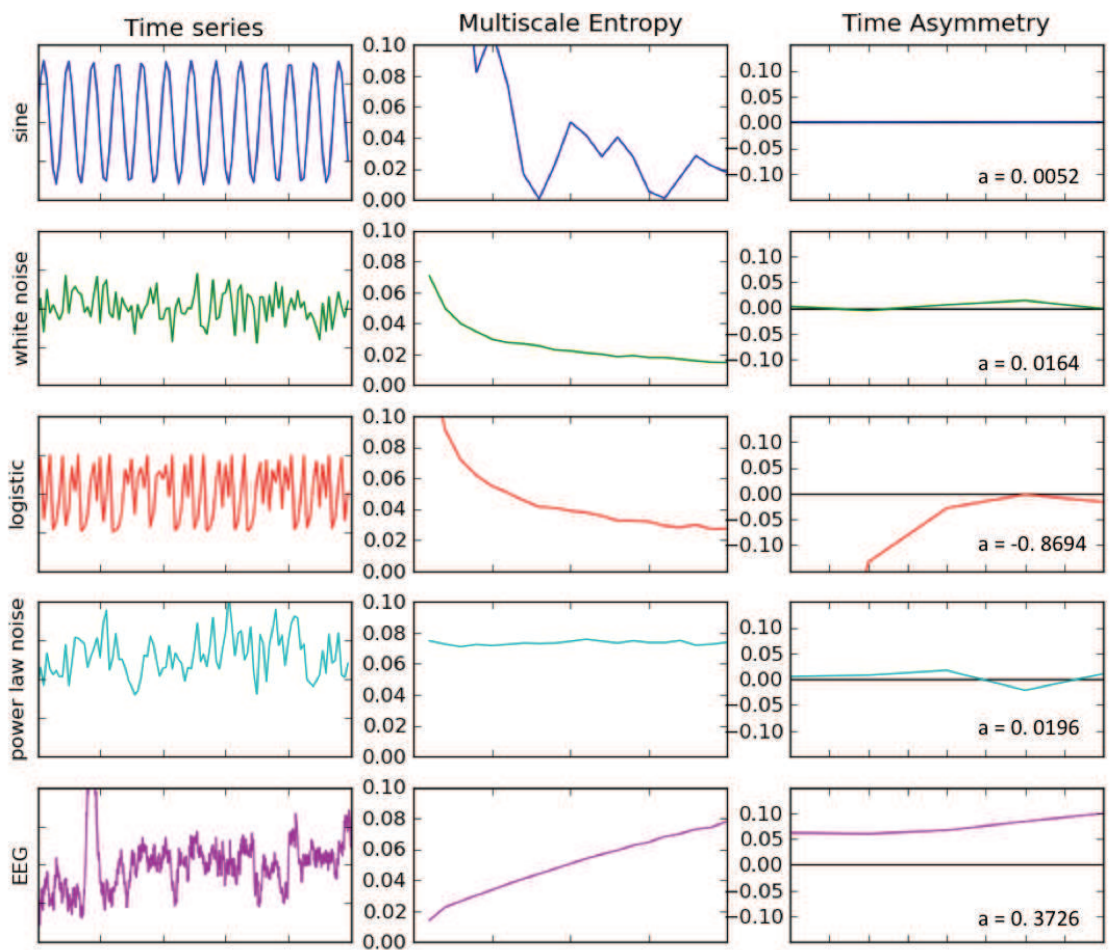

Fig. 1. Characteristics of five different time series are shown. Column 1 shows the time series amplitudes. Column two is the multiscale entropy, where the horizontal axis is the coarse graining scale, from 1 to 20 . Column 3 is the multiscale time asymmetry value. The value of $\mathrm{a}$ in the lower right corner of the time asymmetry plot is the value of the time asymmetry index summed over scales 1 to 5 . A non-zero time asymmetry value is a sufficient condition for nonlinearity of a time series.

After computing multiple resolutions of the EEG time series using the multiscale algorithm shown in equation 2, an estimate of the time irreversibility for each resolution is computed by noting that a symmetric function or time series will have the same number of increments as decrements. That is, the number times $\left|x_{i+1}-x_{i}\right|>0$ will be approximately the same as the number of times $\left|x_{i+1}-x_{i}\right|<0$. Thus, an estimate of the time series symmetry (or reversibility) was found by summing increments and decrements and dividing by the length of the series. A reversible time series will have a value of zero. For a series of 5000 points, as 
used in figure $1, t_{\text {rev }}>0.1$ is a significant indicator of irreversibility and thus of nonlinearity (Schreiber and Schmitz, 1997). This information is used only to indicate that nonlinear information is contained in the EEG time series that is not used in linear analysis methods, suggesting that the MSE may contain more diagnostically useful information than power spectra analysis alone.

Additional methods for characterizing nonlinear signals may be derived from recurrence plot analysis, to be discussed separately below. Communications and electrical engineers may be especially well suited to research in analyzing brain activity and applying methods from communication signals analysis to find information that can be correlated to behavioral and cognitive assessment data. Integration of new results from both nonlinear time series analysis and complex network research may prove to be a fruitful approach for engineers interested in finding patterns in neural activity that are correlated to complex behavioral patterns that psychiatrists and psychologists use to characterize mental health.

\subsection{Synchronization}

While signal complexity is a property of a single time series or EEG channel, transient synchronized activity is a measure of the interaction between different channels and an indication of communication and coordination between different brain regions. Synchronization may be used as a marker for diagnosing underlying mental disorders that involve aberrant long-range connectivity in the brain and may also reveal causal mechanisms (Whittington, 2008). The complexity of synchronization patterns appears to change during network development and reflects different neural wiring schemes and levels of cluster organization (Fuchs et al., 2007).

The synchronization patterns of complex networks have been shown to be closely related to the topology of the network (Arenas et al., 2006) and are related to brain connectivity (Sakkalis et al., 2008). Synchronization between sensors is an indicator of connectivity between brain regions on a scale commensurate with the sensor spacing. EEG signals are believed to derive from pyramidal cells aligned in parallel in the cerebral cortex and hippocampus (Sörnmo and Laguna, 2005), which act as many interacting nonlinear oscillators (Nunez and Srinivasan, 2006). Synchronization between gamma activity (typically defined to be $30-50 \mathrm{~Hz}$ range) is believed to be involved in long-range communication between brain regions. A possible link between gamma activity and the hemodynamic response measured by fMRI was found in a study of auditory response. Distinct activations in the gamma frequency range were found in subcortical structures, including the anterior cingulate cortex (ACC) and thalamus (Mulert et al., 2007).

Synchronized oscillations are transiently stable, thus form and decay rapidly. Synchrony can result from a common input oscillator, such as in cardiac synchronization. It can also be an emergent, self-organized phenomenon that is related to the network structure itself. The latter is of particular relevance to the search for psychiatric biomarkers that are associated with complex behaviors. The complexity of synchronization patterns appears to change during network development and reflects different neural wiring schemes and levels of cluster organization (Fuchs et al., 2007). It is thus reasonable to suppose that the developing brain will show different but characteristic synchronization patterns at different developmental stages. While the fundamental neurophysiological correlates of these patterns may be difficult to ascertain, they nevertheless may serve as a marker for normal and abnormal brain functional development. The emergence of a "social brain network" during early childhood was found in a study of evoked response potentials (ERPs) in 3-, 4-, 
and 12-month-old infants viewing faces of different orientation and direction of eye gaze (Johnson et al., 2005), suggesting a particular pattern of brain connectivity that develops in early childhood. Default mode networks (DMNs) found in adults and (negatively) associated with particular cognitive and sensorimotor activities were lacking in a study of (premature) 3 month old infants (Fransson et al., 2007). However, resting state networks have recently been shown to emerge in the first year of life, suggesting development of brain networks and their potential disruption in neurodevelopmental disorders (Supekar et al.; Uddin et al., 2010).

There is no consensus at this time on the best methods for determining nonlinear synchronization in neurological data and a number of different algorithms have been proposed (Kreuz et al., 2007; Sakkalis et al., 2009). Although strong signal synchronization would likely be detected by any of several nonlinear synchronization measures, two measures that are based on different algorithmic approaches are chosen here (see (Sakkalis et al., 2009) for more thorough discussion of some of the relative merits of each).

Two methods outlined here for computing bivariate synchronization matrix are: (1) the synchronization likelihood (SL) method (Montez et al., 2006); and (2) the instantaneous cross modulation from the circular phase Hilbert transform-based synchronization index (HI), which is robust to signal noise and short time windows (Gans et al., 2009).

Synchronization indices may be searched for correlation in each frequency bands using centered moving averages (Bashan et al., 2008). This approach will find weak or strong correlations with time lags. For each pair, the relative phase index can be computed and stored in a correlation matrix. At each time, some channels may be synchronized. A clustering algorithm can be applied to all channels at a single (averaged) time segment. The result is analogous to gene expression profile clustering (Ramoni et al., 2002). Statistical significance of clusters can be determined by assigning a numerical label to each channel involved in a cluster and the fractional overlap of clusters in different individuals computed. Synchronized clusters may also exhibit very low frequency oscillations, with frequencies of 1 to 0.01 hertz. These have been found in fMRI studies of default mode networks (Broyd et al., 2009; Greicius et al., 2008; Uddin et al., 2010).

Synchronization likelihood (SL) is a method based on the assumption that neurons are highly nonlinear devices, hence methods from chaotic dynamical systems may effectively capture the relevant dynamics of the system (Sakkalis et al., 2009). It is an unbiased generalized synchronization method that relies on detection of simultaneously occurring patterns that may differ in two time series. A method for automatically computing all but two user parameters for the SL algorithm has been developed and will be used here (Montez et al., 2006).

Instantaneous cross modulation (synchronization) of EEG channels can be computed using the Hilbert transform method (Gans et al., 2009). This method is robust to noise and detects synchronization across all frequency bands. The $\mathrm{n}: \mathrm{m}$ cyclic relative phase index $\mathrm{y}_{1,2}$ between two signals, $f_{1}(t)$ and $f_{2}(t)$, at a specific time $t$ is computed over a time interval using a sliding window as:

$$
\Psi_{1,2}^{n, m}(t)=\left|n \phi_{1}(t)-m \phi_{2}(t)\right|, \bmod 1
$$

where $f(t)=\arctan (H(y) / y)$ and $H(y)$ is the Hilbert transform of the time series $y$. This approach is stable for nonstationary data (Gans et al., 2009), which is appropriate for our 
data. The mod 1 term ensures that significant phase differences will be detected even in the presence of noise-induced phase jumps. In most cases $n=m=1$ is commonly assumed, though cross correlation of signals with $\mathrm{n}$ not equal $\mathrm{m}$ will be used here. Two signals are defined to be synchronized when $\Psi_{1,2}$ is less than a specified constant. This algorithm is stable for nonstationary data and will detect synchronization without the need to distinguish between noise and chaos (Gans et al., 2009). A sliding window will be used to compute sync over 5 minutes.

A number of methods have been used for determining synchronization in neurological data and a number of different algorithms have been proposed (Kreuz et al., 2007; Sakkalis et al., 2009). Although useful, many of these methods have difficulties with nonstationary, nonlinear signals and either fail to find true synchrony or introduce spurious synchronization (Fine et al., 2010). Spurious synchronization due to volume conduction effects can be removed by applying a spatial algorithm to ICA decomposition (Hironaga and Ioannides, 2007). ICA eliminates volume conduction effects while maintaining the same time resolution, thus still allowing generalized synchronization to be computed.

As Fourier spectrum can only give meaningful interpretation to linear and stationary processes, its application to data from nonlinear and nonstationary processes is problematical. A relatively new method for extracting instantaneous phase and frequency information from both linear and nonlinear, chaotic signals is the Huang-Hilbert transform (Huang and Wu, 2005; Huang et al., 2009). Determination of instantaneous phase and frequency is usually accomplished using the Hilbert transform method (Kreuz et al., 2007). However, this is only appropriate for monofrequency analytic signals that have a single center of rotation in the complex plane. The Empirical Mode Decomposition (EMD) introduced by Huang makes no assumptions about linearity. The EMF decomposes a nonlinear, nonstationary time series into adaptively determined characteristic time scales of each of the components (Huang et al., 2009). These component functions are termed intrinsic mode functions (IMF) and are analogous to Fourier components in a traditional linear decomposition. The IMFs computed using the empirical mode transform (EMF) have the property of a single center of rotation in the complex plane, ideally satisfying the requirements for the application of the Hilbert transform to determine instantaneous phase and frequency (Fine et al., 2010; Huang et al., 2009).

After computation of IMFs for each EEG channel, the IMF components with the highest power will be used to compute an instantaneous phase coherence matrix, $\mathrm{R}$, using a sliding window. Following (Bialonski and Lehnertz, 2006), R is computed:

$$
R_{i j}=\left(\frac{1}{w} \sum_{t=0}^{w-1} e^{i\left(\phi_{i t}-\phi j t\right.}\right), \bmod 1
$$

where $w$ is the number of time samples in the time series segment or window, $i$ and $j$ designate the channel number (or the IMF component of the channel) and $\mathrm{f}(\mathrm{t})=\arctan (\mathrm{H}(\mathrm{y}) / \mathrm{y})$ and $\mathrm{H}(\mathrm{y})$ is the Hilbert transform of the IMF component. The mod 1 term ensures that significant phase differences will be detected even in the presence of noise-induced phase jumps. The Hilbert transform obtains the best fit of a sinusoid to each IMF at every point in time, identifying an instantaneous frequency (IF), along with its associated instantaneous amplitude (IA). The IF and IA provide a time-frequency decomposition of the data that is highly effective at resolving non-linear and transient features. This algorithm is stable for nonstationary data and will detect synchronization 
without the need to distinguish between noise and chaos (Gans et al., 2009). An example of bivariate synchronization between two EEG sensors in the right medical parietal region is shown in figure 2. The synchronization likelihood in this example was computed using the only the first three IMFs from each sensor, without searching for cross band synchronization.

To identify synchronized clusters of EEG channels, a method based on an eigenvector space method, using eigenvalues of $\mathrm{R}$, can be used, following the algorithm developed and applied in (Allefeld and Bialonski, 2007; Bialonski and Lehnertz, 2006; Fine et al., 2010). The outcome of this algorithm will be synchronized clusters of EEG channels. These may be mapped onto scalp plots and the identified clusters compared to default mode networks that have been identified in young children (Sauseng and Klimesch, 2008; Supekar et al., 2009; Supekar et al., 2010). It will be of particular interest to determine if synchronization clusters are significantly correlated with functional networks in the brain and are biomarkers of abnormalities in brain network function (Assaf et al., 2010; Kennedy and Courchesne, 2008). To date, most research on functional brain networks, including the default mode network, has relied on functional MRI. Networks determined by fMRI reflect only the hemodynamic or metabolic response of neurons (Power et al., 2010). This can be considered a kind of amplitude correlation but not true synchronization of brain regions.

If synchronization of electrical activity can be shown to be an alternative measure of brain network activity it would open up much more exploration of the role of brain networks in cognitive activity, brain computer interfaces and neuropsychiatric disorders. Aberrations to default mode networks have been implicated in a number of brain disorders (Broyd et al., 2009) including post traumatic stress syndrome (Daniels et al., 2010), social phobias (Gentili et al., 2009), depression (Sheline et al., 2009), ADHD (Uddin et al., 2008), autism (Di Martino et al., 2009), and schizophrenia (Lagioia et al., 2010). fMRI is far too expensive to be used routinely as a clinical screening and monitoring tool. Yet the apparent widespread role of synchronized brain networks in many neuropsychiatric disorders suggests that a less expensive and easy to administer technology for analyzing brain networks would be widely useful in clinical practice.

\subsection{Recurrence quantitative analysis}

Several univariate measures of time series complexity and a number of approaches for computing the degree of synchronization between signals have been used to analyze EEG data. Applications of these methods to psychiatric care and mental health continue to show promise. A more general framework for characterizing the dynamics of complex systems may be to construct recurrence plots (Marwan et al., 2007) and compute quantitative properties. The idea to use recurrence plots as a representation of complex system dynamics was first proposed by (Eckmann et al., 1987) in the late 1980s. The original tool presented a graphical means for visualizing differences in system dynamics. Methods for quantifying the small scale structures in recurrence plots were devised and shown to be capable of revealing system parameters and transitions that are not easily obtained by other methods (Marwan et al., 2002; Zbilut et al., 2002). Some dynamical parameters, such as K2 entropy and mutual information can also be derived from recurrence plots without RQA methods by computing the distribution of line lengths in the recurrence plot (Marwan et al., 2007). Readers are referred to the references for reviews of this unifying approach to nonlinear systems analysis. 
(Schinkel et al., 2007) demonstrated that a single measure from RQA analysis could detect a change in the N400 response in single trials when subjects were presented with an oddball task, suggesting that RQA may be a sensitive measure of transient brain states. Few studies have been done using RQA to determine more stable brain functional characteristics. This may be a promising new field for research on EEG biomarkers of psychiatric disorders.

\section{Clinical applications}

\subsection{Infant brain development and autism spectrum disorders}

Autism spectrum disorder (ASD) constitutes a heterogeneous developmental syndrome that is characterized by a triad of impairments that affect social interaction, communication skills, and a restricted range of interests and activities (APA, 2000), with highly variable outcomes. Studies have consistently shown that early intervention leads to better long-term outcomes. But early intervention is predicated on early detection. Behavioral measures have thus far proven ineffective in diagnosing autism before about 18 months of age, in part because the behavioral repertoire of infants is so limited. Neural development may precede overt behavioral observations and thus provide an earlier marker for emerging autistic behaviors. Yet, measuring functional brain development is difficult because few noninvasive methods are available for infants and it is not clear what features to measure that are biomarkers of normal development. As discussed above, multiscale entropy computed from EEG time series has been shown to be a particularly informative analysis tool for physiological signals.

Complex mental disorders such as autism are associated with abnormal brain connectivity that may vary between different regions and different scales (Noonan et al., 2009). Estimation of changes in neural connectivity might be an effective diagnostic biomarker for abnormal connectivity development that leads to ASD behaviors. The electrical signals produced by neural networks are believed to contain information about the network structure (Raghavendra et al., 2009; Stam, 2005; Zavaglia et al., 2008). The physics of complex networks suggests that system invariants will be encoded in the time series produced and measured by EEG sensors (Fuchs et al., 2007; Gao and Jin, 2009). Multiscale entropy (MSE) discussed above is one invariant measure of system dynamics that has been shown to particularly sensitive to changes in physiological systems (Costa et al., 2005b; $\mathrm{Hu}$ et al., 2009b; Takahashi et al., 2010), including mental disorders such as Alzheimer's Disease (Abasolo et al., 2006), schizophrenia (Takahashi et al., 2010), the effect of antipsychotic drugs (Takahashi et al., 2010) and normal aging (Bruce et al., 2009). Preliminary results suggest that MSE may be a biomarker for autism endophenotypes (Bosl et al., 2011) and may provide an earlier diagnosis than behavioral assessments.

MSE values were computed for 79 different infants: 46 at high risk for ASD (hereafter referred to as HRA) based on having an older sibling with a confirmed diagnosis of ASD and 33 controls, defined on the basis of a typically developing older sibling and no family history of neurodevelopmental disorders. The study participants were part of an on-going longitudinal study and for this analysis visits were evaluated at regular intervals. Infants were seated on their mothers' laps in a dimly lit room while a research assistant engaged their attention by blowing bubbles. This procedure was followed to limit the amount of head movement made by the infant that would interfere with the recording process. Continuous EEG was recorded with a 64-channel Sensor Net System. The data were amplified, filtered (band pass $0.1-100.0 \mathrm{~Hz}$ ), and sampled at a frequency of $250 \mathrm{~Hz}$. Data 
were manually reviewed to remove sections with obvious artifacts and continuous, clean 20 second segments were identified to compute alert, resting state MSE values.

Results shown in figure 2 were computing by averaging all channels in a given region, including left/right hemispheres, left/right frontal and total scalp MSE values. The plots are derived by averaging regional MSE values for all infants at a given age. These reveal a significant difference between high risk and typically developing infants (Bosl et al., 2011). The data suggest that there are not only significant differences between the high risk group and typically developing controls, but MSE follows characteristic trajectories during development. To our knowledge, these preliminary results are the first demonstration of a complex systems analysis of EEG data for biomarkers in infants at risk for a complex neurodevelopmental disorder. More details about this study and the use of machine learning to discover diagnostically significant patterns in MSE data can be found in (Bosl et al., 2011).
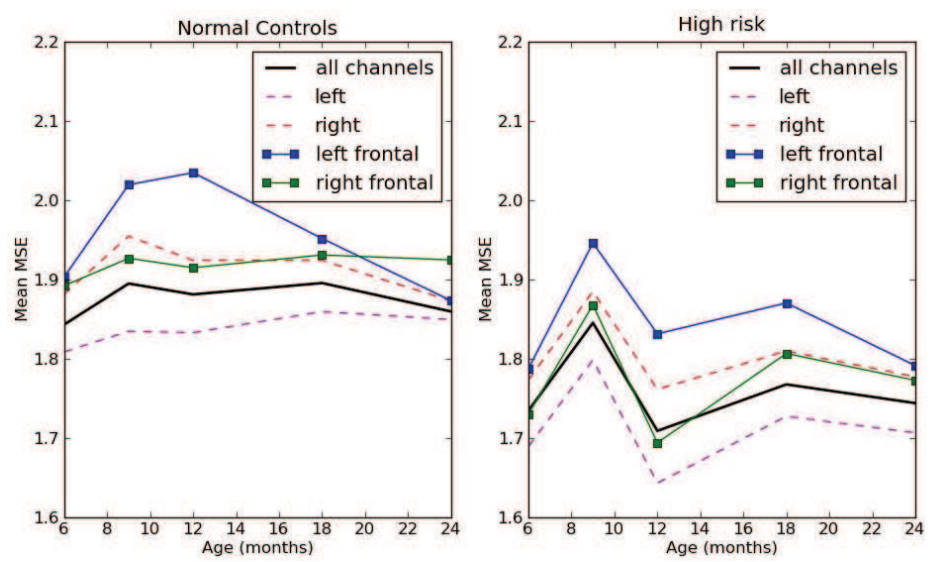

Fig. 2. The change in mean MSE over all channels is shown for each age. Averaging over all channels reveals that in general MSE is higher than the normal controls than in the high risk group, but regional differences cannot be seen.

The trajectory of the curves between 6 and 12 months in figure 2 appear to be as informative as information at any specific age. This is a period of important changes in brain function that are foundational for the emergence of higher level social and communicative skills that are at the heart of ASD. A key characteristic of autism is lack of social perception; autistic spectral disorders are also associated with abnormal brain connectivity (Belmonte et al., 2004; Kleinhans et al., 2008). A number of major cognitive milestones typically occur beginning at around the age 9 months and perhaps earlier in girls. These include joint perception (Behne et al., 2005) and loss of the ability to discriminate certain categories of faces (Pascalis et al., 2002). The latter developments are especially significant because they reveal how socially-grounded experiences influence changes in the neurocognitive mechanisms that underlie speech and face processing. In a prospective study, (Ozonoff et al., 2010) found that social communicative behaviors in infants that later developed ASD declined dramatically between 6 and 18 months when compared to typically developing infants. The results shown here may indicate that MSE is sensitive to neural correlates underlying the observed social and cognitive developments. 
Currently there are no objective medical tests for diagnosing autism. According to the American Academy of Pediatrics (AAP), data strongly suggest better outcomes in children whose conditions are diagnosed early and participate in early intervention programs. Children who are diagnosed early and given intensive behavioral intervention can often be mainstreamed and live normal, productive lives. The AAP recommends that all children be screened for autism at 18 and again at 24 months. Unfortunately, behavioral assessment is time consuming and requires specialized training, both of which pose a problem for routine screening. Because atypical brain development is likely to precede abnormal behavior by months or even years, a critical developmental window for early intervention may be missed if diagnosis is based entirely on a behavioral phenotype. If reliable methods can be developed to lower the age of diagnosis, and if insight is gained into the biological mechanisms that underlie the disorder, it may be possible to develop intervention strategies that can be implemented during the first year of life. Complex systems analysis of EEG may enable a relatively inexpensive and reliable way to estimate the risk of developmental disorders within the first year of life.

\subsection{Schizophrenia}

Early studies of signal synchronization in patients diagnosed with schizophrenia used a linear measure of complexity based on global correlation of all channels (Wackermann et al., 1993) and synchronization based on linear correlation between amplitudes (Saito et al., 1998). Although these methods will necessarily miss nonlinear signal characteristics, they found significant differences between patients and normal controls that were interpreted to reflect decreased coordination between different brain regions, a characteristic of schizophrenia (Uhlhaas et al., 2008; Whittington, 2008).

One interpretation of biological complexity is that it reflects a systems' ability to quickly adapt and function in a changing environment (Costa et al., 2005b). The complexity of EEG signals was found in one study to be associated with the ability to attend to a task and adapt to new cognitive tasks; a significant difference in complexity was found between normal subjects and those with diagnosed schizophrenia (Li et al., 2008). Schizophrenic patients were found to have lower complexity than normal controls in some EEG channels and significantly higher interhemispheric and intrahemispheric cross mutual information values (another measure of signal complexity) than the normal controls (Na et al., 2002).

While signal complexity is a property of a single time series or EEG channel, transient synchronized activity is a measure of the interaction between different channels and an indication of communication and coordination between different brain regions. Synchronization may be used as a marker for diagnosing underlying mental disorders such as schizophrenia and may also reveal causal mechanisms (Whittington, 2008). The complexity of synchronization patterns changes during network development and reflects different neural wiring schemes and levels of cluster organization (Fuchs et al., 2007).

Abnormalities in phase synchronization between multiple bands have been found to be sensitive biomarkers for mental dysfunction in schizophrenic patients (Uhlhaas et al., 2008; Whittington, 2008). Unfortunately, similar abnormalities in synchronous activity have been found associated with a number of other mental disorders (Uhlhaas et al., 2008), so further research is required to discover if more refined patterns of synchrony exist for discriminating different disorders or subtypes. A developmental perspective may be useful here. For example, while many attempts to correlate cortical thickness with intelligence have failed, recent research demonstrated that specific characteristic growth trajectories of cortical 
thickness from infancy to early teen years were highly correlated with above or below average intelligence (Shaw et al., 2006), suggesting that growth curves of brain function may contain more information than any combination of measurements at one specific age. This would require routine brain measurements become part of the medical record and algorithms that recognize abnormal trends would need to be used to interpret data after regular checkups.

\section{Neurotechnology for global mental health}

Mental healthcare in developing nations has long been overlooked by organizations concerned with global health needs. A number of reasons can be cited for this, but one significant factor is the difficulty of accurately diagnosing and classifying mental disorders and the relatively intensive need for human expertise to administer assessments and care. Precision healthcare is a term coined by Clayton Christensen to describe objective medical diagnosis and prescription that is enabled by new technologies. Innovative neurotechnologies that enable basic mental diagnosis to be administered in a cost effective manner in clinics that may be staffed by technicians is urgently needed in developing nations. Even if the diagnostic precision is not as high as might be possible in big city hospital in the developed world, low-cost basic care would serve to alleviate a great deal of personal suffering as well as the economic burden imposed.

The need for neurotechnology to enable precision mental healthcare has never been greater. The developed world is spending greater and greater amounts of money on healthcare and costs threaten their economic vitality. Developing nations will benefit tremendously from even basic neurological and mental healthcare.

While considerable resources have been devoted to finding cures for infectious diseases such as HIV and malaria, relatively little attention has been given to the neurological and cognitive effects of these infections on young patients that recover from the acute infection. A majority of HIV infected patients manifest HIV-associated neurocognitive disorders despite receiving highly active antiretroviral therapy (Van Rie et al., 2009). The developing brains of children who have acquired HIV through vertical infection are particularly vulnerable to central nervous system insults either directly, through secondary infections or from side effects of antiretroviral therapies (LLorente et al., 2009). For example, school age children with perinatally acquired HIV infections receiving antiretroviral therapy displayed lower cognitive function than either HIV affected or HIV unaffected children. Although the antiretroviral therapy reduced HIV symptoms, the cognitive deficits were not improved (Puthanakit et al., 2010).

These clinical observations inform the need to obtain a better understanding of how and when HIV affects cognitive function and to develop novel therapeutic drug candidates to prevent or interfere with progression of HIV associated neurocognitive impairment (Robertson et al., 2010). Some treatments for the primary or secondary infections, may be more harmful to vulnerable brain tissue in children that have been compromised by HIV infection than in adults (LLorente et al., 2009). Research on the effects of HIV infection on the developing brain is a topic of increasing importance as new antiretroviral treatments become more widely available causing many more HIV-1 infections to be treated as a chronic condition to be managed rather than as a fatal disease (Abubakar et al., 2008). Few longitudinal studies have been done of HIV infected children in developing regions who have been receiving antiretroviral therapy. The methods for measuring and analyzing brain 
electrical activity developed here may help to fill the need for objective, cross-cultural measures of cognitive function to estimate the effects of HIV on brain development in children.

It is hoped that neuroengineers will adopt methods outlined in this chapter to discover biomarkers for monitoring the progression of cognitive development and demonstration their utility in the context of HIV associated neurocognitive disability (HAND). Protocols are needed for early assessment and diagnosis of mental and cognitive impairment due to HIV infection, secondary infections and drug therapy. These may also be used as a clinical tool for widespread screening of HIV infected or exposed infants that may enable developmental cognitive impairments to be diagnosed before other HIV symptoms are apparent. Such tools would represent a major step forward in the effort to understand the long-term impact of chronic HIV infection and other infections on cognitive development in children and to provide a new approach for monitoring the cognitive effects of long term management of HIV as a chronic condition in developing regions of the world.

\section{Conclusion}

Neuroscience has been called the next frontier science after the breakthroughs achieved by physics in the mid-twentieth century and genetics and biophysics at the end of the $20^{\text {th }}$ century and early $21^{\text {st }}$ century. The brain is of great scientific interest because of the emergent phenomenon of thought that arises from the vast complexity of this organ. While the question of how the brain thinks is likely to remain a challenging scientific and philosophical topic for a long time, the need for practical innovations to meet the healthcare needs of the world is more urgent. Two issues drive this need at the present time. First, of course, is the tremendous amount of suffering that is the result of brain injury and mental disease. Mental disorders in particular are a difficult challenge for medicine because in most cases there is not a single cause that can easily be diagnosed and "cured". Rather, the perspective throughout this chapter has been that mental disorders are complex emergent phenomena and must therefore be analyzed as such.

This is an exciting and promising time for clinical neuroscience research due to the convergent development of several technologies at this time in history. Although EEG equipment has been around since the 1920s, the creation of inexpensive, wireless, high quality EEG headsets by innovative companies such as Emotiv Systems and Neurosky opens up research possibilities with EEG equipment for many more neuroscientists. As the cost comes down and quality goes up, these tools become practical for routine clinical use. The rapid development of the physics of complex networks and systems, as well as mathematical methods for analyzing complex (chaotic) time series that are produced by complex networks enables new information from the signals measured with inexpensive EEG devices to be extracted and studied as markers for neural correlates of behavior. Finally, the continuing advance of pattern recognition algorithms from the artificial intelligence community enables more subtle pattern-markers to be discovered in complex EEG signal features.

The high cost of healthcare threatens the economies of developed nations and prevents many people in developing regions from obtaining the psychiatric care that they need. On both of these fronts, innovative neurotechnologies for early detection and monitoring of mental disorders are urgently needed. The ready availability of mobile communication devices introduces a platform for making information technology based mental healthcare 
available to many people that have no access at this time. Integration of EEG devices and complex systems methods with clinical decision support tools and mobile device-based health records promises both greatly improved neuropsychiatric healthcare and lower costs. It is hoped that the methods introduced in this chapter will inspire a new generation of bioengineers concerned with mental health to create new tools for clinical neuroscience while closing the gap between neurology and psychiatry.

\section{Acknowledgements}

This research was supported by grants from the National Institute of Mental Health and the Simons Foundation.

\section{References}

Abasolo, D., Hornero, R., Espino, P., Alvarez, D., and Poza, J. (2006). Entropy analysis of the EEG background activity in Alzheimer's disease patients. Physiol Meas 27, 241-253.

Abubakar, A., Van Baar, A., Van de Vijver, F.J., Holding, P., and Newton, C.R. (2008). Paediatric HIV and neurodevelopment in sub-Saharan Africa: a systematic review. Trop Med Int Health 13, 880-887.

Allefeld, C., and Bialonski, S. (2007). Detecting synchronization clusters in multivariate time series via coarse-graining of Markov chains. Phys Rev E Stat Nonlin Soft Matter Phys 76, 066207.

Arenas, A., Diaz-Guilera, A., and Perez-Vicente, C.J. (2006). Synchronization reveals topological scales in complex networks. Phys Rev Lett 96, 114102.

Assaf, M., Jagannathan, K., Calhoun, V.D., Miller, L., Stevens, M.C., Sahl, R., O'Boyle, J.G., Schultz, R.T., and Pearlson, G.D. (2010). Abnormal functional connectivity of default mode sub-networks in autism spectrum disorder patients. Neuroimage 53, 247-256.

Baddeley, R. (2000). Information Theory and the Brain (New York, Cambridge University Press).

Barabasi, A.L. (2009). Scale-free networks: a decade and beyond. Science 325, 412-413.

Bashan, A., Bartsch, R., Kantelhardt, J.W., and Havlin, S. (2008). Comparison of detrending methods for fluctuation analysis. Physica A 387, 5080-5090.

Bassett, D.S., and Bullmore, E. (2006). Small-world brain networks. Neuroscientist 12, 512523.

Bassett, D.S., and Bullmore, E.T. (2009). Human brain networks in health and disease. Curr Opin Neurol 22, 340-347.

Behne, T., Carpenter, M., Call, J., and Tomasello, M. (2005). Unwilling versus unable: infants' understanding of intentional action. Dev Psychol 41, 328-337.

Belmonte, M.K., Allen, G., Beckel-Mitchener, A., Boulanger, L.M., Carper, R.A., and Webb, S.J. (2004). Autism and abnormal development of brain connectivity. J Neurosci 24, 9228-9231.

Bialonski, S., and Lehnertz, K. (2006). Identifying phase synchronization clusters in spatially extended dynamical systems. Phys Rev E Stat Nonlin Soft Matter Phys 74, 051909.

Bosl, W.J. (2000). Modeling Complex Crustal Processes. In GeoComplexity and the Physics of Earthquakes, J.R. Rundle, D.L. Turcotte, and W. Klein, eds. (Washington, D.C., American Geophysical Union). 
Bosl, W.J., Tager-Flusberg, H., and Nelson, C.A. (2011). EEG Complexity as a Biomarker for Autism Spectrum Disorder. BMC Medicine 9.

Braha, D., Minai, A., and Bar-Yam, Y. (2006). Complex engineered systems : science meets technology (Berlin ; New York, Springer).

Broyd, S.J., Demanuele, C., Debener, S., Helps, S.K., James, C.J., and Sonuga-Barke, E.J. (2009). Default-mode brain dysfunction in mental disorders: a systematic review. Neurosci Biobehav Rev 33, 279-296.

Bruce, E.N., Bruce, M.C., and Vennelaganti, S. (2009). Sample entropy tracks changes in electroencephalogram power spectrum with sleep state and aging. J Clin Neurophysiol 26, 257-266.

Buzsáki, G. (2006). Rhythms of the brain (Oxford ; New York, Oxford University Press).

Chen, W., Zhuang, J., Yu, W., and Wang, Z. (2009). Measuring complexity using FuzzyEn, ApEn, and SampEn. Med Eng Phys 31, 61-68.

Costa, M., Goldberger, A.L., and Peng, C.K. (2005a). Broken asymmetry of the human heartbeat: loss of time irreversibility in aging and disease. Phys Rev Lett 95, 198102.

Costa, M., Goldberger, A.L., and Peng, C.K. (2005b). Multiscale entropy analysis of biological signals. Phys Rev E Stat Nonlin Soft Matter Phys 71, 021906.

Costa, M.D., Peng, C.K., and Goldberger, A.L. (2008). Multiscale analysis of heart rate dynamics: entropy and time irreversibility measures. Cardiovasc Eng 8, 88-93.

Cowan, W.M., and Kandel, E.R. (2001). Prospects for neurology and psychiatry. Jama 285, 594-600.

Craddock, R.C., Holtzheimer, P.E., 3rd, Hu, X.P., and Mayberg, H.S. (2009). Disease state prediction from resting state functional connectivity. Magn Reson Med 62, 16191628.

Daniels, J.K., McFarlane, A.C., Bluhm, R.L., Moores, K.A., Clark, C.R., Shaw, M.E., Williamson, P.C., Densmore, M., and Lanius, R.A. (2010). Switching between executive and default mode networks in posttraumatic stress disorder: alterations in functional connectivity. J Psychiatry Neurosci 35, 258-266.

de la Cruz, D.M., Manas, S., Pereda, E., Garrido, J.M., Lopez, S., De Vera, L., and Gonzalez, J.J. (2007). Maturational changes in the interdependencies between cortical brain areas of neonates during sleep. Cereb Cortex 17, 583-590.

Di Martino, A., Ross, K., Uddin, L.Q., Sklar, A.B., Castellanos, F.X., and Milham, M.P. (2009). Functional brain correlates of social and nonsocial processes in autism spectrum disorders: an activation likelihood estimation meta-analysis. Biol Psychiatry 65, 6374.

Dornhege, G. (2007). Toward brain-computer interfacing (Cambridge, Mass., MIT Press).

Douw, L., de Groot, M., van Dellen, E., Heimans, J.J., Ronner, H.E., Stam, C.J., and Reijneveld, J.C. (2010). 'Functional connectivity' is a sensitive predictor of epilepsy diagnosis after the first seizure. PLoS One 5, e10839.

Drongelen, W.v. (2007). Signal processing for neuroscientists : introduction to the analysis of physiological signals (Burlington, Mass., Academic Press).

Eckmann, J., Kaphorst, S.O., and Ruelle, D. (1987). Recurrence plots of dynamical systems. Europhysics Letters 5, 973-977.

Elnashaie, S., and Grace, J.R. (2007). Complexity, bifurcation and chaos in natural and manmade lumped and distributed systems. Chemical Engineering Science 62, 32953325. 
Fine, A.S., Nicholls, D.P., and Mogul, D.J. (2010). Assessing instantaneous synchrony of nonlinear nonstationary oscillators in the brain. J Neurosci Methods 186, 42-51.

Fransson, P., Skiold, B., Horsch, S., Nordell, A., Blennow, M., Lagercrantz, H., and Aden, U. (2007). Resting-state networks in the infant brain. Proc Natl Acad Sci U S A 104, 15531-15536.

Fuchs, E., Ayali, A., Robinson, A., Hulata, E., and Ben-Jacob, E. (2007). Coemergence of regularity and complexity during neural network development. Dev Neurobiol 67, 1802-1814.

Gans, F., Schumann, A.Y., Kantelhardt, J.W., Penzel, T., and Fietze, I. (2009). Crossmodulated amplitudes and frequencies characterize interacting components in complex systems. Phys Rev Lett 102, 098701.

Gao, J. (2007). Multiscale analysis of complex time series : integration of chaos and random fractal theory, and beyond (Hoboken, N.J., Wiley-Interscience).

Gao, J.B., Hu, J., Tung, W.W., and Cao, Y.H. (2006). Distinguishing chaos from noise by scale-dependent Lyapunov exponent. Phys Rev E Stat Nonlin Soft Matter Phys 74, 066204.

Gao, Z., and Jin, N. (2009). Complex network from time series based on phase space reconstruction. Chaos 19, 033137.

Gautama, T., Mandic, D.P., and Van Hulle, M.M. (2003). Indications of nonlinear structures in brain electrical activity. Phys Rev E Stat Nonlin Soft Matter Phys 67, 046204.

Gentili, C., Ricciardi, E., Gobbini, M.I., Santarelli, M.F., Haxby, J.V., Pietrini, P., and Guazzelli, M. (2009). Beyond amygdala: Default Mode Network activity differs between patients with social phobia and healthy controls. Brain Res Bull 79, 409413.

Greicius, M.D., Kiviniemi, V., Tervonen, O., Vainionpaa, V., Alahuhta, S., Reiss, A.L., and Menon, V. (2008). Persistent default-mode network connectivity during light sedation. Hum Brain Mapp 29, 839-847.

Hironaga, N., and Ioannides, A.A. (2007). Localization of individual area neuronal activity. Neuroimage 34, 1519-1534.

Holland, J. (1995). Hidden Order: How Adaptation Builds Complexity (New York, AddisonWesley Publishing Co.).

Hornero, R., Abasolo, D., Escudero, J., and Gomez, C. (2009). Nonlinear analysis of electroencephalogram and magnetoencephalogram recordings in patients with Alzheimer's disease. Philos Transact A Math Phys Eng Sci 367, 317-336.

Hu, J., Gao, J., Tung, W.W., and Cao, Y. (2009a). Multiscale analysis of heart rate variability: a comparison of different complexity measures. Ann Biomed Eng 38, 854-864.

Hu, J., Gao, J., Tung, W.W., and Cao, Y. (2009b). Multiscale Analysis of Heart Rate Variability: A Comparison of Different Complexity Measures. Ann Biomed Eng.

Huang, N.E., and Wu, Z. (2005). An Adaptive Data Analysis Method for nonlinear and Nonstationary Time Series:

The Empirical Mode Decomposition and Hilbert Spectral Analysis (Greenbelt, MD, NASA Goddard Space Flight Center).

Huang, N.E., Wu, Z., Long, S.R., and Arnold, K.C. (2009). On Instantaneous Frequency. Advances in Adaptive Data Analysis 1, 177-229.

Hyman, S.E. (2007). Can neuroscience be integrated into the DSM-V? Nat Rev Neurosci 8 , 725-732. 
Irani, F., Platek, S.M., Bunce, S., Ruocco, A.C., and Chute, D. (2007). Functional near infrared spectroscopy (fNIRS): an emerging neuroimaging technology with important applications for the study of brain disorders. Clin Neuropsychol 21, 9-37.

Johnson, M.H., Griffin, R., Csibra, G., Halit, H., Farroni, T., de Haan, M., Tucker, L.A., Baron-Cohen, S., and Richards, J. (2005). The emergence of the social brain network: evidence from typical and atypical development. Dev Psychopathol 17, 599-619.

Kandel, E.R. (1998). A new intellectual framework for psychiatry. Am J Psychiatry 155, 457469.

Kandel, E.R., Schwartz, J.H., and Jessell, T.M. (2000). Principles of neural science, 4th edn (New York, McGraw-Hill Health Professions Division).

Kennedy, D.P., and Courchesne, E. (2008). The intrinsic functional organization of the brain is altered in autism. Neuroimage 39, 1877-1885.

Kleinhans, N.M., Richards, T., Sterling, L., Stegbauer, K.C., Mahurin, R., Johnson, L.C., Greenson, J., Dawson, G., and Aylward, E. (2008). Abnormal functional connectivity in autism spectrum disorders during face processing. Brain 131, 10001012.

Kreuz, T., Mormann, F., Andrzejak, R., Kraskov, A., and Lehnertz, K. (2007). Measuring synchronization in coupled model systems: A comparison of different approaches. Physica D 225, 29-42.

Kulisek, R., Hrncir, Z., Hrdlicka, M., Faladova, L., Sterbova, K., Krsek, P., Vymlatilova, E., Palus, M., Zumrova, A., and Komarek, V. (2008). Nonlinear analysis of the sleep EEG in children with pervasive developmental disorder. Neuro Endocrinol Lett 29, 512-517.

Kuusela, T.A., Jartti, T.T., Tahvanainen, K.U., and Kaila, T.J. (2002). Nonlinear methods of biosignal analysis in assessing terbutaline-induced heart rate and blood pressure changes. Am J Physiol Heart Circ Physiol 282, H773-783.

Lagioia, A., Van De Ville, D., Debbane, M., Lazeyras, F., and Eliez, S. (2010). Adolescent resting state networks and their associations with schizotypal trait expression. Front Syst Neurosci 4.

Laughlin, R.B. (2005). A different universe : reinventing physics from the bottom down (New York, Basic Books).

Le Van Quyen, M. (2003). Disentangling the dynamic core: a research program for a neurodynamics at the large-scale. Biol Res 36, 67-88.

Li, Y., Tong, S., Liu, D., Gai, Y., Wang, X., Wang, J., Qiu, Y., and Zhu, Y. (2008). Abnormal EEG complexity in patients with schizophrenia and depression. Clin Neurophysiol $119,1232-1241$.

LLorente, A.M., Lopresti, C., and Satz, P. (2009). Neurobehavioral and Neurodevelopmental Sequelae Associated with Pediatric HIV Infection. In Handbook of Clinical Child Neuropsychology, C.R. Reynolds, and E. Fletcher-Janzen, eds. (New York, Springer Science + Business Media), pp. 635-669.

Marwan, N., Romano, M.C., Thiel, M., and Kurths, J. (2007). Recurrence plots for the analysis of complex systems. Physics Reports 438, 237-329.

Marwan, N., Wessel, N., Meyerfeldt, U., Schirdewan, A., and Kurths, J. (2002). Recurrenceplot-based measures of complexity and their application to heart-rate-variability data. Phys Rev E Stat Nonlin Soft Matter Phys 66, 026702. 
Mizuhara, H., Wang, L.Q., Kobayashi, K., and Yamaguchi, Y. (2005). Long-range EEG phase synchronization during an arithmetic task indexes a coherent cortical network simultaneously measured by fMRI. Neuroimage 27, 553-563.

Montez, T., Linkenkaer-Hansen, K., van Dijk, B.W., and Stam, C.J. (2006). Synchronization likelihood with explicit time-frequency priors. Neuroimage 33, 1117-1125.

Muehlemann, T., Haensse, D., and Wolf, M. (2008). Wireless miniaturized in-vivo near infrared imaging. Opt Express 16, 10323-10330.

Mulert, C., Leicht, G., Pogarell, O., Mergl, R., Karch, S., Juckel, G., Moller, H.J., and Hegerl, U. (2007). Auditory cortex and anterior cingulate cortex sources of the early evoked gamma-band response: relationship to task difficulty and mental effort. Neuropsychologia 45, 2294-2306.

Na, S.H., Jin, S.H., Kim, S.Y., and Ham, B.J. (2002). EEG in schizophrenic patients: mutual information analysis. Clin Neurophysiol 113, 1954-1960.

Niedermeyer, E. (2003). The clinical relevance of EEG interpretation. Clinical EEG (electroencephalography) 34, 93-98.

Niedermeyer, E., and Lopes da Silva, F.H. (2005). Electroencephalography : basic principles, clinical applications, and related fields, 5th edn (Philadelphia, Lippincott Williams \& Wilkins).

Noonan, S.K., Haist, F., and Muller, R.A. (2009). Aberrant functional connectivity in autism: evidence from low-frequency BOLD signal fluctuations. Brain Res 1262, 48-63.

Norris, P.R., Stein, P.K., and Morris, J.A., Jr. (2008). Reduced heart rate multiscale entropy predicts death in critical illness: a study of physiologic complexity in 285 trauma patients. J Crit Care 23, 399-405.

Nunez, P.L., and Srinivasan, R. (2006). Electric fields of the brain : the neurophysics of EEG, 2nd edn (New York, Oxford University Press).

Ozonoff, S., Iosif, A.M., Baguio, F., Cook, I.C., Hill, M.M., Hutman, T., Rogers, S.J., Rozga, A., Sangha, S., Sigman, M., et al. (2010). A prospective study of the emergence of early behavioral signs of autism. J Am Acad Child Adolesc Psychiatry 49, 256-266 e251-252.

Pascalis, O., de Haan, M., and Nelson, C.A. (2002). Is face processing species-specific during the first year of life? Science 296, 1321-1323.

Percha, B., Dzakpasu, R., Zochowski, M., and Parent, J. (2005). Transition from local to global phase synchrony in small world neural network and its possible implications for epilepsy. Phys Rev E Stat Nonlin Soft Matter Phys 72, 031909.

Pikovsky, A., Rosenblum, M., and Kurths, J. (2001). Synchronization : a universal concept in nonlinear sciences (Cambridge, Cambridge University Press).

Power, J.D., Fair, D.A., Schlaggar, B.L., and Petersen, S.E. (2010). The development of human functional brain networks. Neuron 67, 735-748.

Puthanakit, T., Aurpibul, L., Louthrenoo, O., Tapanya, P., Nadsasarn, R., Insee-ard, S., and Sirisanthana, V. (2010). Poor cognitive functioning of school-aged children in thailand with perinatally acquired HIV infection taking antiretroviral therapy. AIDS Patient Care STDS 24, 141-146.

Raghavendra, B.S., Dutt, D.N., Halahalli, H.N., and John, J.P. (2009). Complexity analysis of EEG in patients with schizophrenia using fractal dimension. Physiol Meas 30, 795808.

Ramoni, M.F., Sebastiani, P., and Kohane, I.S. (2002). Cluster analysis of gene expression dynamics. Proc Natl Acad Sci U S A 99, 9121-9126. 
Ravasz, E., and Barabasi, A.L. (2003). Hierarchical organization in complex networks. Phys Rev E Stat Nonlin Soft Matter Phys 67, 026112.

Robertson, K., Liner, J., Hakim, J., Sankale, J.L., Grant, I., Letendre, S., Clifford, D., Diop, A.G., Jaye, A., Kanmogne, G., et al. (2010). NeuroAIDS in Africa. J Neurovirol 16, 189-202.

Sabeti, M., Katebi, S., and Boostani, R. (2009). Entropy and complexity measures for EEG signal classification of schizophrenic and control participants. Artif Intell Med 47, 263-274.

Saito, N., Kuginuki, T., Yagyu, T., Kinoshita, T., Koenig, T., Pascual-Marqui, R.D., Kochi, K., Wackermann, J., and Lehmann, D. (1998). Global, regional, and local measures of complexity of multichannel electroencephalography in acute, neuroleptic-naive, first-break schizophrenics. Biol Psychiatry 43, 794-802.

Sakkalis, V., Doru Giurc Neanu, C., Xanthopoulos, P., Zervakis, M.E., Tsiaras, V., Yang, Y., Karakonstantaki, E., and Micheloyannis, S. (2009). Assessment of linear and nonlinear synchronization measures for analyzing EEG in a mild epileptic paradigm. IEEE Trans Inf Technol Biomed 13, 433-441.

Sakkalis, V., Tsiaras, V., Michalopoulos, K., and Zervakis, M. (2008). Assessment of neural dynamic coupling and causal interactions between independent EEG components from cognitive tasks using linear and nonlinear methods. Conf Proc IEEE Eng Med Biol Soc 2008, 3767-3770.

Sauseng, P., and Klimesch, W. (2008). What does phase information of oscillatory brain activity tell us about cognitive processes? Neurosci Biobehav Rev 32, 1001-1013.

Scher, M.S., Ludington-Hoe, S., Kaffashi, F., Johnson, M.W., Holditch-Davis, D., and Loparo, K.A. (2009). Neurophysiologic assessment of brain maturation after an 8-week trial of skin-to-skin contact on preterm infants. Clin Neurophysiol 120, 1812-1818.

Schinkel, S., Marwan, N., and Kurths, J. (2007). Order patterns recurrence plots in the analysis of ERP data. Cogn Neurodyn 1, 317-325.

Schreiber, T., and Schmitz, A. (1997). Discrimination power of measures for nonlinearity in a time series. Phys Rev E Stat Nonlin Soft Matter Phys 55, 5443-5447.

Shannon, C.E., and Weaver, W. (1949). A Mathematical Theory of Communication. Bell System Technical Journal 27, 379-423.

Shaw, P., Greenstein, D., Lerch, J., Clasen, L., Lenroot, R., Gogtay, N., Evans, A., Rapoport, J., and Giedd, J. (2006). Intellectual ability and cortical development in children and adolescents. Nature 440, 676-679.

Sheline, Y.I., Barch, D.M., Price, J.L., Rundle, M.M., Vaishnavi, S.N., Snyder, A.Z., Mintun, M.A., Wang, S., Coalson, R.S., and Raichle, M.E. (2009). The default mode network and self-referential processes in depression. Proc Natl Acad Sci U S A 106, $1942-$ 1947.

Singh, I., and Rose, N. (2009). Biomarkers in psychiatry. Nature 460, 202-207.

Sörnmo, L., and Laguna, P. (2005). Bioelectrical signal processing in cardiac and neurological applications (Amsterdam ; Boston, Elsevier Academic Press).

Stam, C.J. (2005). Nonlinear dynamical analysis of EEG and MEG: review of an emerging field. Clin Neurophysiol 116, 2266-2301.

Stauffer, D. (2006). Biology, sociology, geology by computational physicists, 1st edn (Amsterdam ; Boston, Elsevier).

Supekar, K., Musen, M., and Menon, V. (2009). Development of large-scale functional brain networks in children. PLoS Biol 7, e1000157. 
Supekar, K., Uddin, L.Q., Prater, K., Amin, H., Greicius, M.D., and Menon, V. Development of functional and structural connectivity within the default mode network in young children. Neuroimage 52, 290-301.

Supekar, K., Uddin, L.Q., Prater, K., Amin, H., Greicius, M.D., and Menon, V. (2010). Development of functional and structural connectivity within the default mode network in young children. Neuroimage 52, 290-301.

Takahashi, T., Cho, R.Y., Mizuno, T., Kikuchi, M., Murata, T., Takahashi, K., and Wada, Y. (2010). Antipsychotics reverse abnormal EEG complexity in drug-naive schizophrenia: a multiscale entropy analysis. Neuroimage 51, 173-182.

Uddin, L.Q., Kelly, A.M., Biswal, B.B., Margulies, D.S., Shehzad, Z., Shaw, D., Ghaffari, M., Rotrosen, J., Adler, L.A., Castellanos, F.X., et al. (2008). Network homogeneity reveals decreased integrity of default-mode network in ADHD. J Neurosci Methods 169, 249-254.

Uddin, L.Q., Supekar, K., and Menon, V. (2010). Typical and atypical development of functional human brain networks: insights from resting-state FMRI. Front Syst Neurosci 4, 21.

Uhlhaas, P.J., Haenschel, C., Nikolic, D., and Singer, W. (2008). The role of oscillations and synchrony in cortical networks and their putative relevance for the pathophysiology of schizophrenia. Schizophr Bull 34, 927-943.

Van Rie, A., Dow, A., Mupuala, A., and Stewart, P. (2009). Neurodevelopmental trajectory of HIV-infected children accessing care in Kinshasa, Democratic Republic of Congo. J Acquir Immune Defic Syndr 52, 636-642.

Varela, F., Lachaux, J.P., Rodriguez, E., and Martinerie, J. (2001). The brainweb: phase synchronization and large-scale integration. Nat Rev Neurosci 2, 229-239.

Wackermann, J., Lehmann, D., Dvorak, I., and Michel, C.M. (1993). Global dimensional complexity of multi-channel EEG indicates change of human brain functional state after a single dose of a nootropic drug. Electroencephalogr Clin Neurophysiol 86, 193-198.

Ward, L.M. (2003). Synchronous neural oscillations and cognitive processes. Trends Cogn Sci 7, 553-559.

Whittington, M.A. (2008). Can brain rhythms inform on underlying pathology in schizophrenia? Biol Psychiatry 63, 728-729.

Xie, H.-B., He, W.-X., and Liu, H. (2008). Measuring time series regularity using nonlinear similarity-based sample entropy. Physics Letters A 372, 7140-7146.

Zavaglia, M., Astolfi, L., Babiloni, F., and Ursino, M. (2008). The effect of connectivity on EEG rhythms, power spectral density and coherence among coupled neural populations: analysis with a neural mass model. IEEE Trans Biomed Eng 55, 69-77.

Zbilut, J.P., Thomasson, N., and Webber, C.L. (2002). Recurrence quantification analysis as a tool for nonlinear exploration of nonstationary cardiac signals. Med Eng Phys 24, 53-60.

Zhang, D., Ding, H., Liu, Y., Zhou, C., and Ye, D. (2009). Neurodevelopment in newborns: a sample entropy analysis of electroencephalogram. Physiol Meas 30, 491-504. 


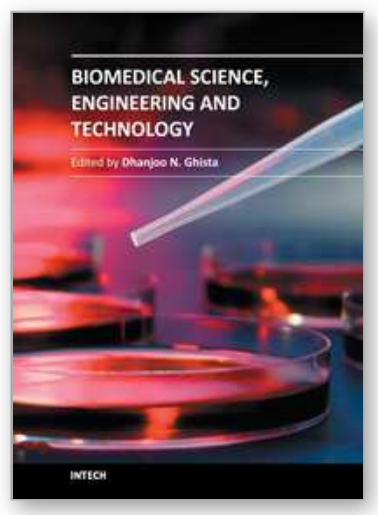

\author{
Biomedical Science, Engineering and Technology \\ Edited by Prof. Dhanjoo N. Ghista
}

ISBN 978-953-307-471-9

Hard cover, 902 pages

Publisher InTech

Published online 20, January, 2012

Published in print edition January, 2012

This innovative book integrates the disciplines of biomedical science, biomedical engineering, biotechnology, physiological engineering, and hospital management technology. Herein, Biomedical science covers topics on disease pathways, models and treatment mechanisms, and the roles of red palm oil and phytomedicinal plants in reducing HIV and diabetes complications by enhancing antioxidant activity. Biomedical engineering coves topics of biomaterials (biodegradable polymers and magnetic nanomaterials), coronary stents, contact lenses, modelling of flows through tubes of varying cross-section, heart rate variability analysis of diabetic neuropathy, and EEG analysis in brain function assessment. Biotechnology covers the topics of hydrophobic interaction chromatography, protein scaffolds engineering, liposomes for construction of vaccines, induced pluripotent stem cells to fix genetic diseases by regenerative approaches, polymeric drug conjugates for improving the efficacy of anticancer drugs, and genetic modification of animals for agricultural use. Physiological engineering deals with mathematical modelling of physiological (cardiac, lung ventilation, glucose regulation) systems and formulation of indices for medical assessment (such as cardiac contractility, lung disease status, and diabetes risk). Finally, Hospital management science and technology involves the application of both biomedical engineering and industrial engineering for cost-effective operation of a hospital.

\title{
How to reference
}

In order to correctly reference this scholarly work, feel free to copy and paste the following:

William J. Bosl (2012). Neurotechnology and Psychiatric Biomarkers, Biomedical Science, Engineering and Technology, Prof. Dhanjoo N. Ghista (Ed.), ISBN: 978-953-307-471-9, InTech, Available from: http://www.intechopen.com/books/biomedical-science-engineering-and-technology/neurotechnology-andpsychiatric-biomarkers

\section{INTECH}

open science | open minds

\author{
InTech Europe \\ University Campus STeP Ri \\ Slavka Krautzeka 83/A \\ 51000 Rijeka, Croatia \\ Phone: +385 (51) 770447 \\ Fax: +385 (51) 686166 \\ www.intechopen.com
}

\author{
InTech China \\ Unit 405, Office Block, Hotel Equatorial Shanghai \\ No.65, Yan An Road (West), Shanghai, 200040, China \\ 中国上海市延安西路65号上海国际贵都大饭店办公楼 405 单元 \\ Phone: +86-21-62489820 \\ Fax: +86-21-62489821
}


(C) 2012 The Author(s). Licensee IntechOpen. This is an open access article distributed under the terms of the Creative Commons Attribution 3.0 License, which permits unrestricted use, distribution, and reproduction in any medium, provided the original work is properly cited. 